\title{
Thermal Conductivity and Interconnectivity of Hexamethylene Diisocyanate Contained Polyurethane Grafted Multiwall Carbon Nanotube/Polyurethane Nanocomposite Film
}

\author{
Sungjin Yun, Hyungu Im and Jooheon Kim \\ School of Chemical Engineering \& Materials Science, Chung-Ang University, Seoul 156-756, Korea
}

\begin{abstract}
The hexamethylene diisocyanate (HDI) contained polyurethane (PU) grafted multi-walled carbon nanotubes (HDI-g-MWNTs) have been synthesized by simple blending method to fabricate thermal conductive nanocomposite. This method shows that HDI-g-MWNTs can improve the interfacial compatibility between HDI-g-MWNTs and matrix. The HDI base PU thermal conductive film (HDI-PU) showed enhanced dispersibility of functionalized MWNTs because of low crystallinity which was affected by its steric hindrance. The length of MWNTs was prevented until the end of the reaction. The long distance of MWNTs could be help to make good thermal conducting path. These phenomena have influence on the excellent thermal conductivity. The thermal conductivity increased from $\kappa=0.274 \mathrm{~W} / \mathrm{mK}$ to $\kappa=0.645 \mathrm{~W} / \mathrm{mK}$, as the addition of the MWNTs increase to $1.2 \mathrm{vol} \%$. The interconnectivities of HDI-Polyurethane have higher value than other composite films. [doi:10.2320/matertrans.M2010322]
\end{abstract}

(Received September 15, 2010; Accepted December 2, 2010; Published January 26, 2011)

Keywords: carbon nanotube, polyurethane, thermal conductivity, interconnectivity, hexamethylene diisocyanate

\section{Introduction}

Carbon nanotubes (CNTs) have been the focus of extensive research efforts in the context of multifunctional nanocomposite materials. The MWNTs have excellent aspect ratios of 100-1000 high specific surface areas, and thermal conductivity reported to be $3000 \mathrm{~W} / \mathrm{mK}$ at room temperature. ${ }^{1,2)}$ The thermal conductivity of MWNTs is higher than that of conventional thermal conductors such as diamond and graphite. ${ }^{1)}$

Polyurethane (PU) is an important class of polymer materials that find use in a variety of applications owing to its useful properties such as excellent flexibility, elasticity, and good adhesion. ${ }^{3,4)}$ A method widely used to improve its thermal conductivity is the dispersion of inorganic fillers in the PU matrix. ${ }^{5)}$

Previous research on the thermal properties of MWNTs/ PU composites involved a variety of methods. Cai et al. reported a composite made by inserting a solution containing pristine MWNTs and sodium dodeecyl sulphate (SDS) into a polycaprolactone (PCL)-based PU matrix; the thermal conductivity thus achieved was $0.47 \mathrm{~W} / \mathrm{mK}^{6)} \mathrm{Xia}$ et al. dispersed MWNTs in polyol containing BYK9077 (dispersing agent, Blagden Specialty Chemicals Ltd., England); then, this solution yielded a MWNTs/PU (methylene diphenyl diisocyanate, MDI) composite. The thermal conductivity of the film prepared by this method was $0.19 \mathrm{~W} / \mathrm{mKK}^{7)}$

Previous methods have shown that MWNTs do not exhibit high dispersibility in a PU matrix. Therefore, in this study, we synthesized HDI-g-MWNT thermal conducting film via a simple blending method to improve compatibility between HDI-g-MWNTs and a HDI-based matrix. The thermal conductivity of our composite film is considerably higher than those of the composite films reported in previous studies; the interconnectivity of HDI-g-MWNTs in the HDIbased matrix is also higher than that of other composites.

\section{Experimental Procedure}

Pristine MWNTs $(0.2 \mathrm{~g})$ were functionalized by refluxing them with $40 \mathrm{ml}$ of a mixture of $98 \mathrm{vol} \%$ sulfuric acid $\left(\mathrm{H}_{2} \mathrm{SO}_{4}\right)$ and $70 \mathrm{vol} \%$ nitric acid $\left(\mathrm{HNO}_{3}\right)(3: 1$ by vol\%, respectively). The reaction mixture was stirred at $323 \mathrm{~K}$ for $24 \mathrm{~h}$ and then added to deionized water. The homogenous suspension thus obtained was filtered through a 450-nm nylon membrane and the filtrate was washed several times. The obtained MWNTs-COOH powders were dispersed in a solution of thionyl chloride $\left(\mathrm{SOCl}_{2}\right)(250 \mathrm{ml})$ by sonication for $2 \mathrm{~h}$ and then stirring for $12 \mathrm{~h}$ at $333 \mathrm{~K}$. Thereafter, the suspension was vacuum-filtered through a 450 -nm polytetrafluoroethylene (PTFE) membrane and vacuum-dried for $12 \mathrm{~h}$ at ambient temperature. The dried MWNTs-COCl powders were added to excess hexamethylenediamine (HMDA) in tetrahydrofuran (THF) under magnetic stirring for $12 \mathrm{~h}$ at $343 \mathrm{~K}$. After amine treatment, the filtrate cake was washed with excess THF; then, the filtrate cake was desiccated overnight at $314 \mathrm{~K}$ in a vacuum oven to yield MWNTs- $\mathrm{NH}_{2}$. This MWNTs- $\mathrm{NH}_{2}$ was then dispersed in $250 \mathrm{ml}$ of $\mathrm{N}, \mathrm{N}$ dimethylformamide (DMF), which was subjected to stirring while $2 \mathrm{~mol}$ of isocyanate (HDI) was dissolved in it. After sonication for $30 \mathrm{~min}$, polyethylene glycol (PEG) with an average molecular weight of 1450 (1 mol) (PEG 1450) and 1,4-buthanediol (BD) (1.5 g) were added to the suspension. The PU-grafted MWNTs were dispersed by stirring for $12 \mathrm{~h}$ and then, this homogenous solution was reacted at $343 \mathrm{~K}$. The HDI-g-MWNTs in the solution were filtered through a nylon membrane and the filtrate was washed twice with DMF. After washing, these HDI-g-MWNTs were dried in a vacuum oven for $4 \mathrm{~h}$ at $323 \mathrm{~K}$. A schematic of this entire reaction procedure is shown in Fig. 1.

$\mathrm{BD}$ and PEG 1450 were mixed in DMF by stirring until a homogeneous solution was formed. This solution was mechanically stirred for $3 \mathrm{~h}$ at $343 \mathrm{~K}$. Thereafter, isocyanates 

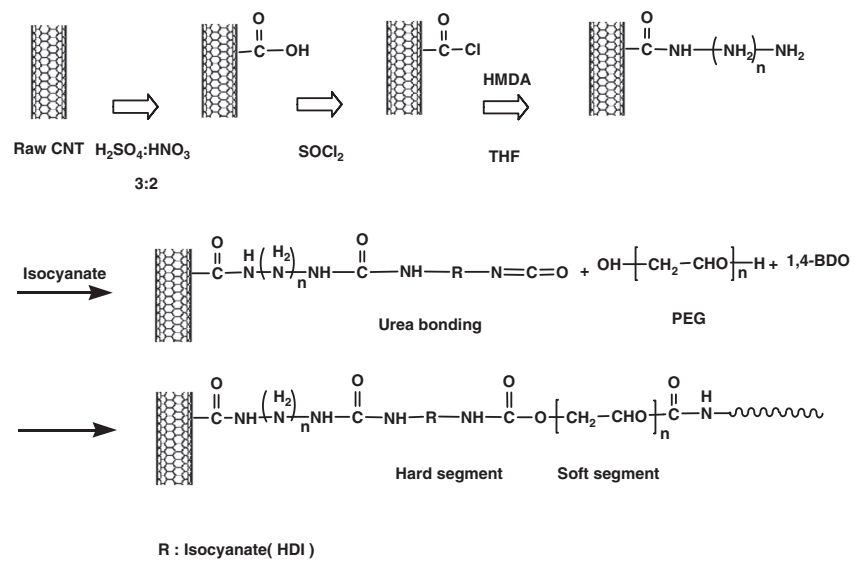

Fig. 1 Chemical route for preparation of HDI-g-MWNTs.

were added to each PU matrix batch, and the temperature was increased to $358 \mathrm{~K}$; the solution was then mechanically stirred for a further $12 \mathrm{~h}$. The prepared HDI-g-MWNTs were dispersed in the PU matrix by mechanical stirring coupled with sonication for $2 \mathrm{~h}$. The amount of HDI-g-MWNTs in the suspension was controlled to obtain composites with different weight fraction. The HDI-PU solution was directly cast by solvent drying on a casting mold (PTFE) to allow slow evaporation of the solvent at $373 \mathrm{~K}$. Approximately $1 \mathrm{ml}$ of the HDI-PU solution was poured into each mold. The thicknesses of the cast films were about $50 \mu \mathrm{m}$. The films were obtained from all mixing solutions and these were dried in a vacuum oven to remove residue solvent.

\section{Results and Discussion}

The linkages between the MWNTs and the PU chains were characterized using FT-IR. The spectra of the pristine and acid-treated MWNTs are shown in Figs. 2(a) and 2(b), respectively. Compared with the pristine MWNTs, the acidtreated MWNTs (A-MWNTs) show two clear broad absorption bands at $3400 \mathrm{~cm}^{-1}$ and $1727 \mathrm{~cm}^{-1}$. The former can be identified as the $\mathrm{O}-\mathrm{H}$ stretching, which is characteristic of carboxyl acid groups $(\mathrm{COOH})$; the latter can be attributed to acid carbonyl $(\mathrm{C}=\mathrm{O})$ stretching. These findings indicated that carboxyl acid groups were attached to the CNTs. The FT-IR spectra of the HDI-g-MWNTs are shown in Fig. 2(c). Two new peaks appeared at around $2900 \mathrm{~cm}^{-1}$ and $1100 \mathrm{~cm}^{-1}$. The broad peak between 2850 and $2950 \mathrm{~cm}^{-1}$ is associated with the stretching vibrations of $\mathrm{C}-\mathrm{H}$ in the alkane chain, and the peak around $1100 \mathrm{~cm}^{-1}$ is associated with the $\mathrm{C}-\mathrm{N}$ stretching of aliphatic amines. The characteristic $\mathrm{O}-\mathrm{H}$ peak observed at about $3600 \mathrm{~cm}^{-1}$ in Fig. 2(c) may have resulted from residual A-MWNTs and $\mathrm{H}_{2} \mathrm{O}$ molecules.

The initial weight loss of the A-MWNTs occurred from approximately $472 \mathrm{~K}$ and continued up to $873 \mathrm{~K}$. The weight loss of the A-MWNTs was about 20 mass $\%$ and it was attributed to the thermal decomposition of the carboxylic acid groups. The composition of the grafted PU can be estimated by calculating the difference between the residual weights of the A-MWNTs and the HDI-g-MWNTs. Figure 3(b) reveals that the HDI-g-MWNTs showed considerably greater weight

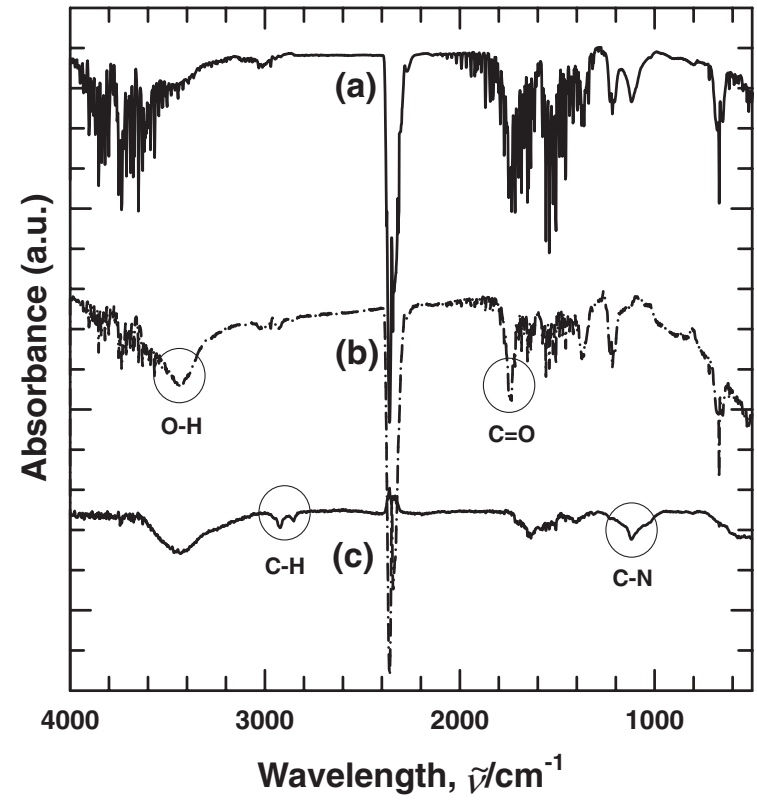

Fig. 2 FT-IR spectra of (a) Pristine MWNTs, (b) A-MWNTs and (c) HDIg-MWNTs.

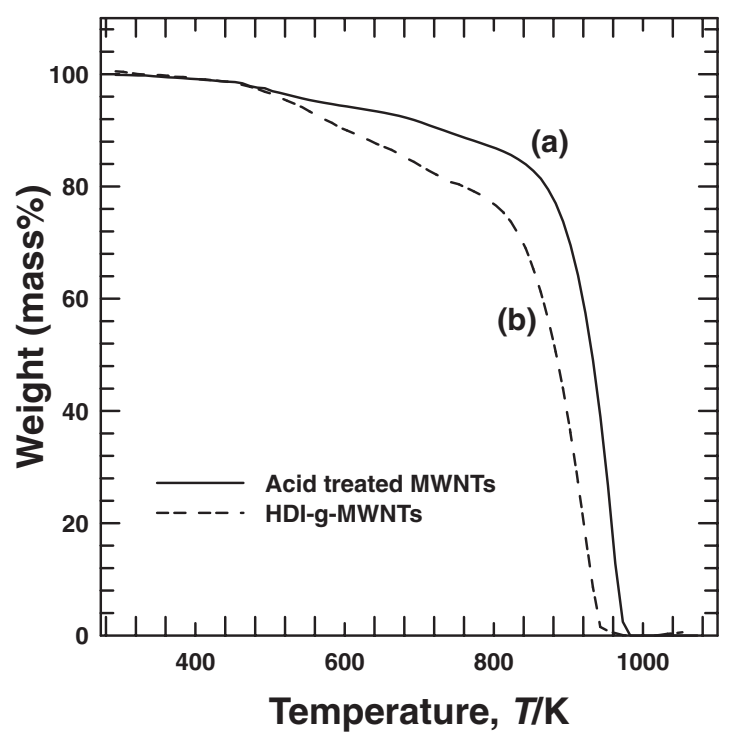

Fig. 3 TGA curves of the (a) Acid treated MWNTs and (b) HDI-gMWNTs.

losses than the A-MWNTs-the difference being about 10 mass $\%$ at approximately $773 \mathrm{~K}$.

Figure 4 shows the morphology of the pristine MWNTs and the HDI-g-MWNTs. The SEM images of the HDI-gMWNTs demonstrate that their surfaces are rougher and thicker than those of the pristine MWNTs. This indicates that the surfaces of the HDI-g-MWNTs were covered with PU. The rod length remained almost the same until the end of all the steps in the process. This result implies that the MWNTs were not damaged during the chemical process.

Figure 4 shows the distribution of the HDI-g-MWNTs on the surface of the HDI-PU film containing 1 mass\% HDI-gMWNTs in the PU matrix. As can be seen in this figure, in the HDI-PU film, the HDI-g-MWNTs were homogeneously dispersed throughout the matrix. 

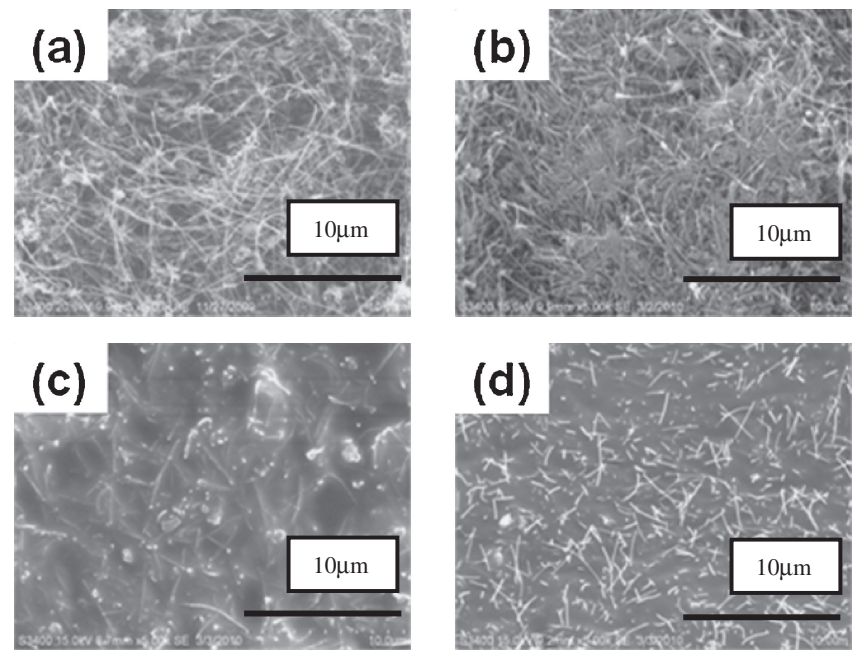

Fig. 4 The SEM images (5.0k magnifications) of (a) Pristine MWNTs, (b) HDI-g-MWNTs, (c) HDI-PU (Surface) and (d) HDI-PU (CrossSection).

The explanation for these observations is related to the crystallinity of PU. The characteristics of incompatibility of alternating segments and microphase separation in PU are well known. ${ }^{8)}$ Therefore, the steric hindrance of the diisocyanate in a hard segment may affect the crystallization of the urethane segment. A previous study conducted by Gaymans et al. ${ }^{8)}$ explained the different crystalline behavior of PU with hard segments. According to this study, steric hindrance arising from the hard segments is related to the phenyl ring. By means of differential scanning calorimetry (DSC) and dynamic mechanical analysis (DMA), Gaymans et al. confirmed that HDI-PU not only showed a high degree of crystallinity but also that the crystallites had a high aspect ratio, as compared to the MDI-based and the 2,4-toluene diisocyanate (TDI)-based polymers.

These differences in the crystallinity of PU also affect MWNTs dispersion. Brooks et al. reported that matrix crystallinity affected the dispersibility of CNTs. ${ }^{9)}$ They indicated that MWNTs are poorly dispersed in low crystallizable polymers. As they explained, the crystallinity of the PU matrix provided the dispersion conditions of CNTs. The high crystallinity of HDI-based PU served to the uniform dispersion of CNTs after sonication in solution. Also, the growth of a semicrystalline structure from the CNTs acts as a barrier and prevents CNT clustering during the casting process. $^{9)}$

The thermal properties of the composite films were measured by the laser flash method to determine the thermal diffusivity and thermal conductivity. The thermal conductivity was calculated using the following equation: ${ }^{10)}$

$$
\kappa=\alpha \times \rho \times C_{\mathrm{p}},
$$

where $\kappa$ is the thermal conductivity of the films, $\alpha$ is the thermal diffusivity, $\rho$ is the density, and $C_{\mathrm{p}}$ is the specific heat capacity under constant pressure. The plot of thermal conductivity vs. volume fraction from 0 to $1.2 \mathrm{vol} \%$ functionalized MWNTs is shown as Fig. 5. Note that for the same volume fraction of MWNTs, the thermal conduc-

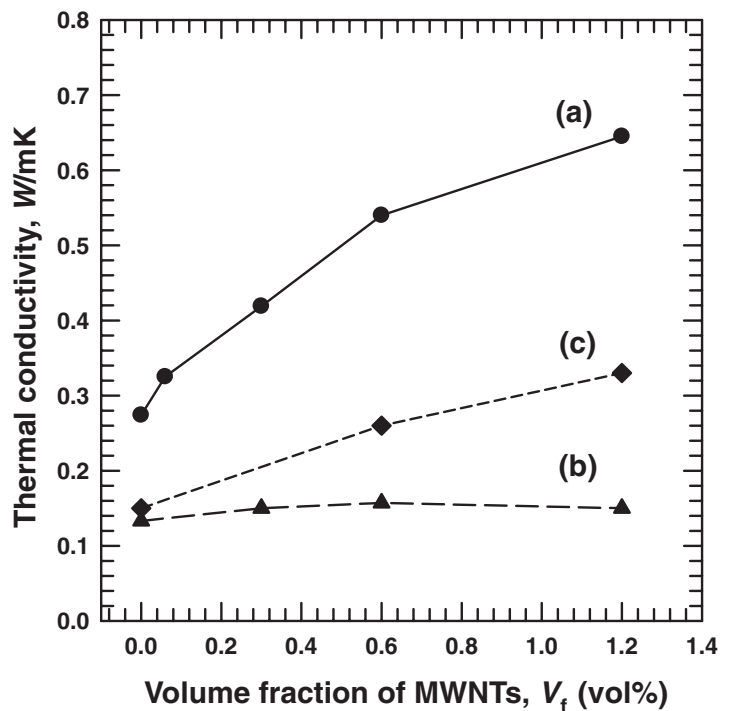

Fig. 5 The thermal conductivity of (a) HDI-PU film, (b) MWNTs(BYK9907)/PU and (c) MWNTs(SDS)/PU.

tivity of HDI-PU films prepared by the method described in this paper was two times and four times greater than the those of the films prepared in previous studies by Cai et al. ${ }^{6)}$ and Xia et al. ${ }^{7)}$ respectively.

The calculated thermal interconnectivity, shown in Fig. 6 and 7 , could aid the determination of the degree of dispersibility of HDI-g-MWNTs in a PU matrix. To obtain the value of thermal interconnectivity, we calculated, as follows, the Hashin-Shtrikman lower (2) and upper boundaries (3). ${ }^{11,12)}$

$$
\begin{aligned}
\sigma_{\mathrm{comp}, 1} & =\sigma^{\mathrm{HS}-} \\
\sigma_{\mathrm{comp}, 1} & =\sigma_{\mathrm{pol}} \frac{2 \sigma_{\mathrm{pol}}+\sigma_{\mathrm{fill}}-2 x_{\mathrm{fill}}\left(\sigma_{\mathrm{pol}}-\sigma_{\mathrm{fill}}\right)}{2 \sigma_{\mathrm{pol}}+\sigma_{\mathrm{fill}}+x_{\mathrm{fill}}\left(\sigma_{\mathrm{pol}}-\sigma_{\mathrm{fill}}\right)} \\
\sigma_{\mathrm{comp}} & =\sigma^{\mathrm{HS}+} \\
\sigma_{\mathrm{comp}} & =\sigma_{\text {fill }} \frac{2 \sigma_{\mathrm{fill}}+\sigma_{\mathrm{pol}}-2 x_{\mathrm{pol}}\left(\sigma_{\mathrm{fill}}-\sigma_{\mathrm{pol}}\right)}{2 \sigma_{\mathrm{fill}}+\sigma_{\mathrm{pol}}+x_{\mathrm{pol}}\left(\sigma_{\mathrm{fill}}-\sigma_{\mathrm{pol}}\right)}
\end{aligned}
$$

where $\sigma_{\text {pol }}, \sigma_{\text {Fill }}$ and $x_{\text {fill }}$ represent the conductivity of polymer matrix, the conductivity of filler, and the volume fraction of filler, respectively. The measured thermal conductivities were located between the upper boundary and lower boundary, as shown in Fig. 6. The thermal conductivities of HDI-PU were close to the lower boundary because the conducting HDI-g-MWNTs particles are surrounded by the matrix. Schilling and Partzsch suggested the following formula for calculating the interconnectivity in the conducting phase $\left(X_{\text {interconnectivity }}\right)^{13)}$

$$
X_{\text {interconnectivity }}=\frac{\sigma_{\text {measured }}-\sigma^{\mathrm{HS}-}}{\sigma^{\mathrm{HS}+}-\sigma^{\mathrm{HS}-}}
$$

As can be seen from Fig. 7, the interconnectivities of the HDI-PU film prepared in this study are the higher than those of the films prepared in previous studies. ${ }^{6,7)}$ This high interconnectivity of HDI-PU implies that the proportion of MWNTs in the PU matrix is high and that these MWNTs are well interconnected. These phenomena show that HDIPU films formed better thermal conducting paths than other 


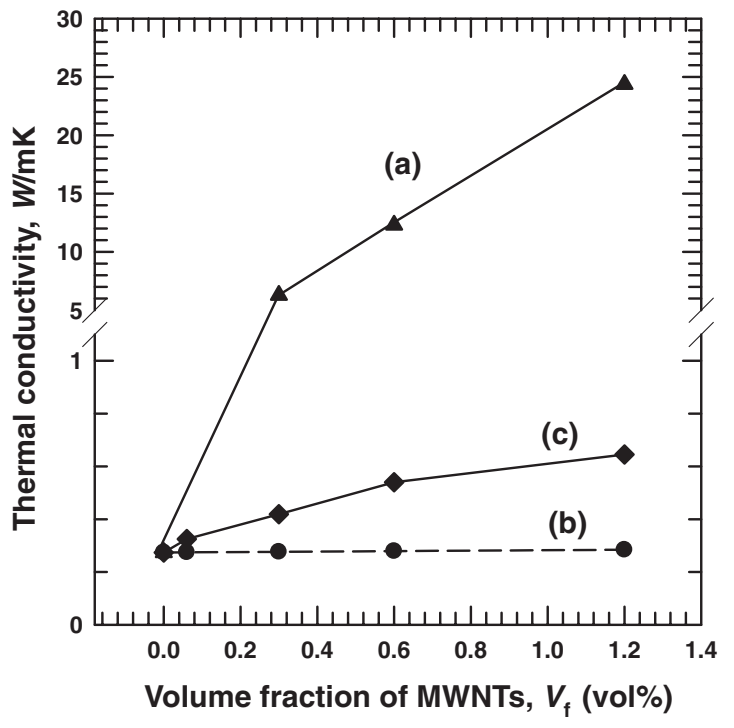

Fig. 6 The thermal conductivities of H-PU films compared to the HashinShtrikman (a) Upper and (b) lower boundaries and (c) the thermal conductivity of HDI-PU film.

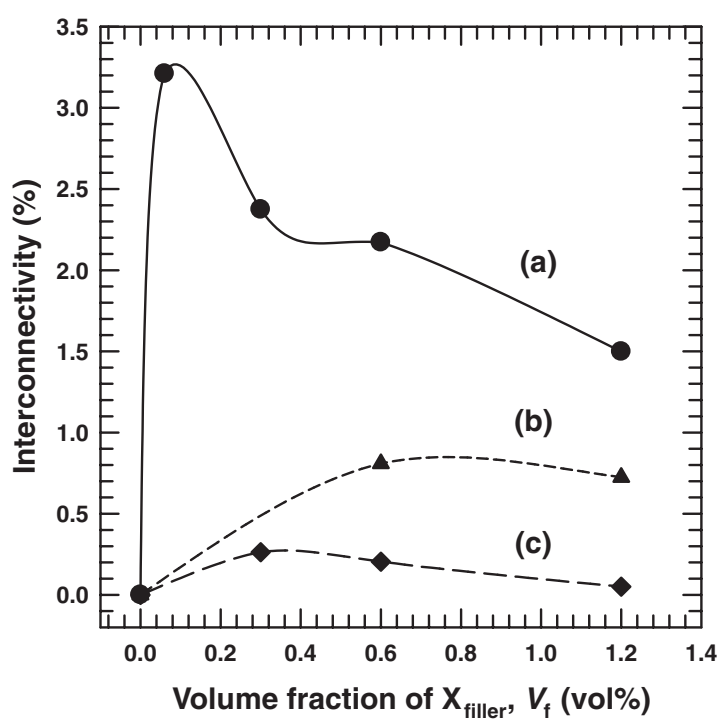

Fig. 7 Interconnectivities of (a) HDI-PU film, (b) MWNTs(BYK9907)/ PU and (c) MWNTs(SDS)/PU.

MWNTs/PU composite films. ${ }^{6,7)}$ The interconnectivity of the HDI-PU film at a high volume fraction of MWNTs decreased because of the HDI-g-MWNTs aggregated in the PU matrix.

\section{Conclusion}

HDI-g-MWNTs/PU composite films were successfully synthesized using a simple blending method. The synthesized HDI-g-MWNTs show excellent compatibility with the HDIPU matrix. The dispersibility of the HDI-g-MWNTs in the HDI-PU matrix was affected by hard segment structures. The linear structure of HDI-PU has high crystallinity, and this structure could aid the dispersion of HDI-g-MWNTs in the HDI-PU matrix during the casting process. This phenomenon affected the excellent thermal conductivity of HDI-PU. A maximum thermal conductivity of $0.645 \mathrm{~W} / \mathrm{mK}$ was obtained at approximately $1.2 \mathrm{vol} \%$. The thermal conductivities at the upper and lower boundaries were calculated using the formula derived by Hashin-Shtrikman; these values were then used to determine the interconnectivity of the conducting phase in HDI-PU. The interconnectivities and thermal conductivity of the HDI-PU film prepared in this study are higher than other MWNTs/PU composite films. This result indicates that HDI-g-MWNTs were well dispersed in the HDI-PU matrix and that the interconnectivity in the conducting phase was good.

\section{Acknowledgement}

This research was supported by a grant from the Fundamental R\&D Program for Core Technology of Materials funded by the Ministry of Knowledge Economy, Republic of Korea.

\section{REFERENCES}

1) C. H. Liu, H. Huang, Y. Wu and S. S. Fan: Appl. Phys. Lett. 84 (2004) 4248-4250.

2) M. A. Osman and D. Srivastava: Nanotechnology 12 (2001) 21-24.

3) Y. L. Du and T. C. Wen: Mater. Chem. Phys. 71 (2001) 62-69.

4) S. D. Lee, O. J. Kwon, B. C. Chun, J. W. Cho and J. S. Park: Fiber Polymer 10 (2009) 71-76.

5) H. Xia and M. Song: J. Mat. Chem. 16 (2006) 1843-1851.

6) D. Cai and M. Song: Carbon 46 (2008) 2107-2112.

7) H. Xia and M. Song: Soft Matter 1 (2005) 386-394.

8) G. J. E. Biemond, K. Braspenning and R. J. Gaymans: J. Appl. Polym. Sci. 107 (2008) 2180-2189.

9) K. Joen, S. Warnock, C. Ruiz-orta, A. Kismarahardja, J. Brooks and R. Alamo: J. Polym. Sci.: Part B 48 (2010) 2084-2096.

10) H. S. Kim, Y. S. Chae, B. H. Park, J. S. Yoon, M. S. Kang and H. J. Jin: Current Appl. Phys. 8 (2008) 803-806.

11) F. R. Schiling and G. M. Partzsch: Phys. Chem. Earth (A) 26 (2001) 239-246.

12) X. Lu and G. Xu: J. Appl. Polym. Sci. 65 (1997) 2733-2738.

13) S. Yu, P. Hing and X. Hu: Comp. Part A 33 (2002) 289-292. 